UWThPh-2004-24

UAB-FT-570

\title{
Violation of a Bell inequality in particle physics experimentally verified?
}

\author{
R.A. Bertlmann ${ }^{\text {a }}$, A. Bramon ${ }^{\text {b }}$, G. Garbarino ${ }^{\text {c }}$, B.C. Hiesmayr ${ }^{\text {a }}$ \\ ${ }^{a}$ Institut für Theoretische Physik, Boltzmanngasse 5, A-1090 Vienna, Austria \\ ${ }^{\mathrm{b}}$ Grup de Física Teòrica, Universitat Autònoma de Barcelona, E-08193 Bellaterra, \\ Spain \\ ${ }^{\mathrm{c}}$ Dipartimento di Fisica Teorica, Università di Torino and INFN, Sezione di \\ Torino, I-10125 Torino, Italy
}

\begin{abstract}
Relevant aspects for testing Bell inequalities with entangled meson-antimeson systems are analyzed. In particular, we argue that the results of A. Go, J. Mod. Optics 51, 991 (2004), which nicely illustrate the quantum entanglement of $B$-meson pairs, cannot be considered as a Bell-test refuting local realism.
\end{abstract}

Key words: Entanglement, Bell inequality, $B$-meson system, Neutral kaon system PACS: 03.65.Ud, 14.40.-n

\section{Introduction}

A recent paper [1] reports that the famous Bell inequality [2] has been tested in a high-energy physics experiment for the first time. Data on entangled $B$-mesons, produced via the resonance decay $\Upsilon(4 S) \rightarrow B^{0} \bar{B}^{0}$ at the KEKB asymmetric $e^{+} e^{-}$collider and collected at the BELLE detector, were analyzed. Events of EPR-entangled $B^{0} \bar{B}^{0}$ meson pairs, identified via their semileptonic decays, were used to claim for a violation of a Bell inequality (BI) in the version of Clauser, Horne, Shimony and Holt $(\mathrm{CHSH})[3]$ by more than $3 \sigma$. In this Letter we analyze the relevant circumstances for testing Bell inequalities with entangled meson-antimeson systems and, consequently, the significance of the reported result.

Though the authors widely appreciate the interest in basic questions of quantum mechanics (QM) explored in particle physics, they have to argue that 
the reported result is scarcely relevant in the discussion of a violation of the Bell inequality for the entangled $B$-meson system. In the authors opinion, the proof of the existence of correlations which are stronger than those explainable by a theory based on the assumptions of locality and realism is not conclusive due to the following two main drawbacks:

(1) "Active" measurements, opening the possibility to choose among alternative setups, are missing; in other words, there is no free choice for the experimenter on the the specific question asked to the system.

(2) The time evolution of an unstable quantum state is unitary only if the state for the decay products is included. The "information" of these decay products cannot be ignored as done in Ref. [1].

In addition, the authors will briefly review recent proposals on how to test the peculiar non-local correlations predicted by quantum theory for EPRentangled massive systems in high-energy physics. Most of these proposals refer to two-kaon systems coming from $\phi(1020)$-resonance decays or protonantiproton annihilations, quite similar to the process $\Upsilon(4 S) \rightarrow B^{0} \bar{B}^{0}$ considered in Ref. [1].

In 1935 Einstein, Podolsky and Rosen [4] claimed to have shown that quantum mechanics was an incomplete theory. Their reasoning relied on two assumptions - realism and locality - and on a precise criterion for completeness. The introduction of local hidden variables, which complement the information contained in conventional state vectors, would allow for such a completion of QM. In 1964 John S. Bell [2] considered the whole class of completions with local hidden parameters and showed that all expectation values or probabilities derived within that class are constrained to obey certain inequalities. However, expectation values or probabilities derived within QM can contradict these Bell inequalities. With this milestone, Bell shifted the original arguments of Einstein, Podolsky and Rosen about the physical reality of quantum systems from the realm of philosophy to the domain of experimental testing. Moreover, a whole new field which is now of increasing interest was also opened. Indeed, the EPR-entanglement is the basic ingredient of new technologies such as quantum information and quantum communication (see, e.g., Ref. [5]).

In the last two decades we have witnessed an outstanding progress in testing the peculiar correlations predicted by quantum theory between outcomes of space-like separated measurements. But a decisive and loophole-free experiment, which would rule out any local realistic theory (LRT), has not been yet performed in the opinion of the authors (for a different view, see Refs. [6]). Notwithstanding, the authors want to stress that they believe - as it is also the firm consensus in the community - that there is almost no doubt that the outcomes of this type of experiments will agree with QM. The goal of such discussions and experiments is then to re-educate our intuition and to 
understand the very principles of quantum theory such that one will be able to use them for new technologies.

The deficiencies of experiments testing Bell inequalities are essentially twofold and usually known as the "locality" and the "detection efficiency" loophole. The Weihs et al. experiment [7] with entangled photons closes the first loophole but not the second one. Conversely, the Rowe et al. experiment [8] with entangled beryllium ions closes the second loophole but not the one related to locality. This locality loophole requires space-like separated measurements by the two observers, Alice and Bob, i.e., alternative measurement settings that can be changed sufficiently fast during the flight of the two particles and that can be chosen completely at will or (more easily) at random on each side. The detection efficiency loophole arises from the low efficiency of the detectors only a small subset of all produced pairs is detected, most of the pairs are lost. One is then forced to introduce the additional and non-testable fair sampling hypothesis assuming that the reduced set of detected events behaves like the total set.

Finally, Hasegawa et.al [9] reported an experiment with single neutrons in an interferometric device which shows a violation of a Bell-like inequality. The entanglement is achieved not between two separate particles but between two degrees of freedom of a single neutron, namely, between the path it takes in the interferometer and its spin component which is different for the two paths. The mathematical description of the entangled state is the same as for the previously mentioned systems. However, as there are no two spatially separated particles, it is contextuality rather than non-locality that is tested (see also Ref. [10]).

\section{Requirements for testing a Bell inequality and drawbacks of Ref.[1]}

\subsection{Choice arguments}

Generally, when discussing Bell inequalities and, particularly, in the CHSHversion, Alice can choose to measure either with setup $A$ or $A^{\prime}$, each one having two possible outcomes. Similarly, Bob can choose between his two dichotomic setups $B$ or $B^{\prime}$. By her choice, the particles under Alice's control are projected either onto the $A$ - or the $A^{\prime}$-basis, but the measurement outcome in the chosen basis is, of course, under God's dices and out of Alice's control. The fact of being able to choose "actively" between alternative bases is crucial to derive Bell inequalities from local realism. This possibility of a choice is strictly needed in order to argue that "...had Alice chosen in the very last moment to 
measure $A$ instead of $A^{\prime}$, her choice would not modify the outcome of Bob's measurement...". As it is well known [11], counterfactuality, as exemplified in the above sentence, is necessary in deriving a genuine Bell inequality: without an "active" choice it is indeed impossible to enforce the locality condition.

For the previously mentioned experiments with photons, ions or neutrons, such an "active" choice between alternative measurement bases with dichotomic outcomes was clearly possible. But the situation is different in the $B$-meson experiment [1]. In this case, one starts with the $B^{0} \bar{B}^{0}$ state

$$
\begin{aligned}
\left|\psi^{-}(0)\right\rangle & =\frac{1}{\sqrt{2}}\left\{\left|B^{0}\right\rangle_{l} \otimes\left|\bar{B}^{0}\right\rangle_{r}-\left|\bar{B}^{0}\right\rangle_{l} \otimes\left|B^{0}\right\rangle_{r}\right\} \\
& \simeq \frac{1}{\sqrt{2}}\left\{\left|B_{L}\right\rangle_{l} \otimes\left|B_{H}\right\rangle_{r}-\left|B_{H}\right\rangle_{l} \otimes\left|B_{L}\right\rangle_{r}\right\}
\end{aligned}
$$

where $l$ and $r$ denote the "left" and "right" directions of motion of the two separating $B$-mesons and $\left|B_{L, H}\right\rangle=\left\{\left|B^{0}\right\rangle \pm\left|\bar{B}^{0}\right\rangle\right\} / \sqrt{2}$, once (small) $\mathcal{C} \mathcal{P}$ violation effects are ignored. One then allows for their time evolution in free space given by

$$
\left|B_{L, H}\right\rangle \rightarrow e^{-i m_{L, H} t} e^{-\frac{1}{2} \Gamma_{L, H} t}\left|B_{L, H}\right\rangle
$$

where $\Gamma_{L} \simeq \Gamma_{H}=\Gamma_{B}=1 / \tau_{B}(\hbar=1)$ is the common decay width of the light$\left(m_{L}\right)$ and heavy-mass $\left(m_{H}\right)$ eigenstates, $B_{L}$ and $B_{H}$, of the non-Hermitian, "effective-mass" Hamiltonian. The mass difference, $\Delta m \equiv m_{H}-m_{L}$, induces $B^{0}-\bar{B}^{0}$ oscillations in time, detectable by $B$-meson flavor measurements with quantum number "beauty" $B=+1$ for $B^{0}$ and $B=-1$ for $\bar{B}^{0}$. This requires the discrimination of the $B^{0}$ decay modes from their corresponding charge conjugate modes from $\bar{B}^{0}$, e.g., $B^{0} \rightarrow D^{*-} l^{+} \nu$ vs $\bar{B}^{0} \rightarrow D^{*+} l^{-} \bar{\nu}$, as done in Ref. [1]. As explained in Refs. [12,13,14], the different times $t_{A}, t_{A^{\prime}}, \ldots$ (Alice's side) and $t_{B}, t_{B^{\prime}}, \ldots$ (Bob's) of the joint flavor measurements play then the same role as the distinct orientations of the polarization analyzers in photonic experiments. The procedure is formally analogous to that in the above mentioned experiments, but it is by no means an "active" measurement. There is no way for the experimenter to force a $B$-meson to decay at a given instant $t_{A}$ or $t_{A^{\prime}}$, i.e., she/he cannot choose "actively" the measurement bases and the decay just occurs according to the well-known probabilistic law. It is Nature that decides the measurement bases leaving no room for counterfactual considerations. Thus, a basic condition for the correct derivation from LRT of the BI used in Ref. [1] is not fulfilled and the results, despite providing a notable test of the QM correlations exhibited by $B^{0} \bar{B}^{0}$ entangled pairs, cannot be relevant when confronting LRT vs QM. 


\subsection{Unitarity constraints}

But, even if flavor measurements could be actively induced at different times, another drawback affects these kind of Bell-tests. This second drawback, originated by the postulate of QM according to the evolution of a closed quantum system is unitary, is a little more involved but not less important. It requires the discussion of the time evolution of the meson states, including the possibility of the decay, a case we certainly do not have to consider for photons or stable spin- $-\frac{1}{2}$ particles. In this paragraph we will explain why the normalization of the expectation value to the surviving meson pairs, Eq. (9) of Ref. [1], is not appropriate. Note that without this normalization no violation of the Bell-CHSH inequality occurs for reasons we discuss in the following.

Quite generally, we consider now decaying neutral meson systems such as $B^{0} \bar{B}^{0}$ or $K^{0} \bar{K}^{0}$ pairs. Because of unitarity of the time evolution, the norm of the total state must be conserved. This means that the decrease of the norm of the meson state must be compensated by the increase of the decay product state norm. Thus we describe the complete time evolution of a meson quantum state through a unitary operator $U(t, 0)$ as follows (see Refs. $[14,15,16]$ )

$$
\left|M_{1,2}\right\rangle \longrightarrow\left|M_{1,2}(t)\right\rangle=U(t, 0)\left|M_{1,2}\right\rangle=e^{-i \lambda_{1,2} t}\left|M_{1,2}\right\rangle+\left|\Omega_{1,2}(t)\right\rangle,
$$

where $\left|M_{1,2}\right\rangle$ represents the eigenstate of the non-Hermitian, "effective mass" Hamiltonian and can be written as superpositions of the flavor states $\left|M^{0}\right\rangle$ and $\left|\bar{M}^{0}\right\rangle$. The exponential evolution of the decaying meson state is given by the eigenvalues $\lambda_{1,2}=m_{1,2}-\frac{i}{2} \Gamma_{1,2}$, with $m_{1,2}$ the mass and $\Gamma_{1,2}$ the decay width of the meson $M_{1,2}$. The state $\left|\Omega_{1,2}(t)\right\rangle$ represents the decay products.

Starting then with an entangled $M^{0} \bar{M}^{0}$ pair, the unitary time evolution also provides a contribution from the decay product states. These introduce a third possible experimental outcome which complicates the issue because Bell$\mathrm{CHSH}$ inequalities refer to dichotomic measurements only.

For decaying systems, it is therefore crucial to formulate the experimental dichotomic question in accordance with unitarity. The appropriate question on the system when it has evolved up to time $t$ is "Are you a meson $M^{0}$ of a certain flavor $f=+1$ or not?" - question I. It is clearly different to the question "Are you a meson $M^{0}$ with flavor $f=+1$ or an antimeson $\bar{M}^{0}$ with $f=-1$ ?" - question II- as treated in Ref. [1], since all decay products (an additional information from the quantum system) are ignored by the latter. Question I admits just two answers, question II is dichotomic only if conditioned to the survival of both mesons. 
Let us be more concrete and consider the expectation values for a series of correlation measurements in these two cases:

(i) For question II: "Are you a meson $M^{0}$ or an antimeson $\bar{M}^{0}$ ?"

$$
E^{\text {non-unitary }}\left(t_{l} ; t_{r}\right)=-\cos (\Delta m \Delta t) \cdot e^{-\Gamma\left(t_{l}+t_{r}\right)}
$$

where $\Delta m=m_{1}-m_{2}, \Delta t=t_{l}-t_{r}$ and $\Gamma=\left(\Gamma_{1}+\Gamma_{2}\right) / 2$.

(ii) For question I: "Are you a meson $M^{0}$ or not?"

$$
\begin{aligned}
& E^{\text {unitary }}\left(t_{l} ; t_{r}\right)=-\cos (\Delta m \Delta t) \cdot e^{-\Gamma\left(t_{l}+t_{r}\right)} \\
& \quad+\frac{1}{2}\left(1-e^{-\Gamma_{1} t_{l}}\right)\left(1-e^{-\Gamma_{2} t_{r}}\right)+\frac{1}{2}\left(1-e^{-\Gamma_{2} t_{l}}\right)\left(1-e^{-\Gamma_{1} t_{r}}\right) .
\end{aligned}
$$

The second expectation value, Eq. (5), compared to the first one where the decay components are ignored, contains additional terms which express the characteristic contribution coming from the decay product states $\left|\Omega_{1,2}(t)\right\rangle$.

The expectation values of any LRT have to satisfy the following Bell-CHSH inequality [3]:

$$
S=\left|E\left(t_{A} ; t_{B}\right)-E\left(t_{A} ; t_{B^{\prime}}\right)\right|+\left|E\left(t_{A^{\prime}} ; t_{B}\right)+E\left(t_{A^{\prime}} ; t_{B^{\prime}}\right)\right| \leq 2 .
$$

However, the calculation of $S$ using quantum mechanical expectation values shows a critical dependence on the ratio $x=\Delta m / \Gamma$, which can be formulated in the following way (see also Refs. $[14,15,16]$ ).

Proposition: The unitary expectation values (5) do not violate the Bell-CHSH inequality for any choice of the four involved times iff $x=\Delta m / \Gamma<N_{I}$; the non-unitary ones (4) do not violate the inequality iff $x<N_{I I}$.

$N_{I}, N_{I I}$ are bounds which we determine numerically. The values are: $N_{I}=$ 2.6 and $N_{I I}=2.0$ for the $B^{0} \bar{B}^{0}, D^{0} \bar{D}^{0}$ and $B_{s}^{0} \bar{B}_{s}^{0}$ systems, while for the $K^{0} \bar{K}^{0}$ system we have $N_{I} \approx N_{I I}=2.0$ since we can neglect the width of the long-lived K-meson as compared to the short-lived one, $\Gamma_{L} \ll \Gamma_{S}$, implying $E^{\text {non-unitary }}\left(t_{l} ; t_{r}\right) \approx E^{\text {unitary }}\left(t_{l} ; t_{r}\right)$. 
The experimental $x$ values for different meson systems are the following ones:

\begin{tabular}{|r|c|}
\hline \multicolumn{1}{c|}{$x$} & meson system \\
\hline 0.77 & $B^{0} \bar{B}^{0}$ \\
0.95 & $K^{0} \bar{K}^{0}$ \\
$<0.03$ & $D^{0} \bar{D}^{0}$ \\
$>20.60$ & $B_{s}^{0} \bar{B}_{s}^{0}$ \\
\hline
\end{tabular}

Therefore, no violation of the Bell-CHSH inequality occurs for the familiar meson-antimeson systems; only for the last system a violation is expected.

Résumé: Normalizing the non-unitary expectation value (4) to the surviving pairs, $E_{\mathrm{R}}^{\text {non-unitary }}\left(t_{l} ; t_{r}\right)=-\cos (\Delta m \Delta t) / \cosh (\Delta \Gamma \Delta t / 2)$, with $\Delta \Gamma=\Gamma_{1}-\Gamma_{2}$, as in Refs. [1], one obtains a formal violation of the Bell-CHSH inequality. But this is hardly relevant for testing LRT versus QM. The reasons are twofold: Firstly, "active" measurements are missing, therefore an essential hypothesis for the derivation of a genuine Bell inequality is not satisfied; secondly, the unitary time evolution of the unstable quantum state - the decay property of the meson - is ignored, which is part of its Nature. Therefore one has to use the unitary formula (5), which, however, does not lead to a violation of the Bell-CHSH inequality for the familiar systems: $B^{0} \bar{B}^{0}, K^{0} \bar{K}^{0}, D^{0} \bar{D}^{0}$.

\section{Outlook}

It turns out that quantum mechanical tests of meson-antimeson systems are more subtle than naively expected and one has to involve other features of the mesons, which are characteristic for such massive quantum systems, like $\mathcal{C P}$ violation or regeneration of quantum states. For example, neutral kaons exhibit $\mathcal{C P}$ violation in $K^{0} \bar{K}^{0}$ mixing. It is remarkable that $\mathcal{C P}$ violation is connected with the violation of a BI for different $K^{0}-\bar{K}^{0}$ superpositions (i.e., different quasi-spin states instead of different times) of neutral kaons $[14,17,18]$.

It is also quite interesting that, using the well known regeneration mechanism of kaons, novel Bell inequalities can be established [13,19] and tested with $K^{0} \bar{K}^{0}$ pairs produced at $\Phi$-factories and $p \bar{p}$-machines.

Finally, we would like to point out that meson-antimeson systems allow for other tests of QM. A possible approach to investigate the nature of entanglement is to experimentally determine the decoherence of entangled meson 
pairs $[20,21,22]$ and thus the validity of QM. It turns out that decoherence is strikingly connected to the entanglement loss of common entanglement measures [22], e.g., the entanglement of formation or the concurrence. Moreover, other subtle features of quantum mechanics such as quantum erasers [23], quantitative duality [24] or quantitative complementarity [25] are interesting phenomena of meson systems, which have been studied recently.

Acknowledgements: This work has been supported by EURIDICE HPRNCT-2002-00311, INFN and Spanish MCyT, BFM-2002-02588.

\section{References}

[1] A. Go, J. Mod. Optics 51, 991 (2004).

[2] J.S. Bell, Physics 1, 195 (1964).

[3] J.E. Clauser, M.A. Horne, A. Shimony, and R.A. Holt, Phys. Rev. Lett. 23, 880 (1969).

[4] A. Einstein, B. Podolsky and N. Rosen, Phys. Rev. 47, 777 (1935).

[5] R. A. Bertlmann, A. Zeilinger (eds.): Quantum [Un] speakables, from Bell to Quantum Information, Springer, Berlin Heidelberg New York (2002).

[6] A. Aspect, Nature 398, 189 (1999); P. Grangier, Nature 409, 774 (2001).

[7] G. Weihs, T. Jennewein, C. Simon, H. Weinfurter, and A. Zeilinger, Phys. Rev. Lett. 81, 5039 (1998).

[8] M. A. Rowe, D. Kielpinski, V. Meyer, C.A. Sackett, W.M. Itano, C. Monroe, and D.J. Wineland, Nature 409, 791 (2001).

[9] Y. Hasegawa, R. Loidl, G. Badurek, M. Baron, and H. Rauch, Nature 425, 45 (2003).

[10] R.A. Bertlmann, K. Durstberger, Y. Hasegawa and B.C. Hiesmayr, Phys. Rev. A 69, 032112 (2004).

[11] M. Redhead, Incompleteness, nonlocality and realism, Oxford University Press, Oxford (1990).

[12] N. Gisin and A. Go, Am. J. Phys. 69, 264 (2001).

[13] A. Bramon and M. Nowakowski, Phys. Rev. Lett. 83, 1 (1999).

[14] R.A. Bertlmann and B.C. Hiesmayr, Phys. Rev. A 63, 062112 (2001).

[15] B.C. Hiesmayr, Ph.D. thesis, University of Vienna, 2002. 
[16] G.C. Ghirardi, R. Grassi and R. Ragazzon, in The DAФNE Physics Handbook, edited by L. Maiani, G. Pancheri and N. Paver (Servizio Documentazione dei Laboratori Nazionale di Frascati, Italy, 1992), Vol. I, p. 283.

[17] F. Uchiyama, Phys. Lett. A 231, 295 (1997).

[18] R.A. Bertlmann, W. Grimus and B.C. Hiesmayr, Phys. Lett. A 289, 21 (2001).

[19] A. Bramon and G. Garbarino, Phys. Rev. Lett. 88, 040403 (2002); A. Bramon and G. Garbarino, Phys. Rev. Lett. 89, 160401 (2002); B. Ancochea, A. Bramon and M. Nowakowski, Phys. Rev. D 60094008 (1999).

[20] R.A. Bertlmann, W. Grimus, and B.C. Hiesmayr, Phys. Rev. D 60, 114932 (1999).

[21] R.A. Bertlmann and W. Grimus, Phys. Rev. D 64, 056004 (2001).

[22] R.A. Bertlmann, K. Durstberger and B.C. Hiesmayr, Phys. Rev. A 68, 012111 (2003).

[23] A. Bramon, G. Garbarino and B.C. Hiesmayr, Phys. Rev. Lett. 92, 020405 (2004); A. Bramon, G. Garbarino and B.C. Hiesmayr, Phys. Rev. A 69, 062111 (2004).

[24] A. Bramon, G. Garbarino and B.C. Hiesmayr, Eur. J. Phys. C 32, 377 (2004).

[25] A. Bramon, G. Garbarino and B.C. Hiesmayr, Phys. Rev. A 69, 022112 (2004). 